Tohoku Math. J.

66 (2014), 155-169

\title{
LOCAL MAXIMAL FUNCTIONS AND OPERATORS ASSOCIATED TO LAGUERRE EXPANSIONS
}

\author{
Pablo Viola and Beatriz ViViani
}

(Received March 9, 2012, revised April 16, 2013)

\begin{abstract}
In this paper we get sharp conditions on a weight $v$ which allow us to obtain some weighted inequalities for a local Hardy-Littlewood Maximal operator defined on an open set in the Euclidean $n$-space. This result is applied to assure a pointwise convergence of the Laguerre heat-diffusion semigroup $u(x, t)=(T(t) f)(x)$ to $f$ when $t$ tends to zero for all functions $f$ in $L^{p}(v(x) d x)$ for $p$ greater than or equal to 1 and a weight $v$. In proving this we obtain weighted inequalities for the maximal operator associated to the Laguerre diffusion semigroup of the Laguerre differential operator of order greater than or equal to 0. Finally, as a by-product, we obtain weighted inequalities for the Riesz-Laguerre operators.
\end{abstract}

1. Introduction and main results. In this paper we study weighted problem for local maximal operators defined on an open set $\Omega$ in $\boldsymbol{R}^{n}$. The weighted problem is the following:

For a linear or sublinear operator $T$, find conditions on a weight $v$ which assure the existence of a weight $u$ such that $T$ maps $L^{p}(\Omega, v(x) d x)$ into $L^{p}(\Omega, u(x) d x)$.

First, we establish the notation and the general background. Let us consider the setting of $\boldsymbol{R}^{n}$ with Lebesgue measure $m$. If $A$ is a measurable set, we write $|A|$ for $m(A)$. The cube with center $x$ and semidiagonal $r$ will be denoted by $Q(x, r)$. In such a case, its diameter will be $\operatorname{diam}(Q(x, r))=2 r$. We have $|Q(x, r)|=a_{n} r^{n}$, where $a_{n}:=\left(2 n^{-1 / 2}\right)^{n}$. Let $\Omega$ be an open set in $\boldsymbol{R}^{n}$ and suppose the complementary set $F=\boldsymbol{R}^{n} \backslash \Omega$ is nonempty. For a positive constant $\gamma<1 / 4$, consider the family of cubes

$$
\mathcal{F}_{\gamma}=\left\{Q(x, r) ; x \in \Omega, r>0 \text { with } \frac{\gamma \operatorname{dist}(Q(x, r), \partial F)}{r}>1\right\},
$$

where $\partial F$ is the common boundary of $F$ and $\Omega$, and $\operatorname{dist}(Q, \partial F)$ denotes the distance between $Q$ and $\partial F$. Now, we define the local maximal Hardy-Littlewood function $\mathcal{M}_{\mathcal{F}_{\gamma}}$ as

$$
\mathcal{M}_{\mathcal{F}_{\gamma}} f(x):=\sup _{Q \in \mathcal{F}_{\gamma}, x \in Q} \frac{1}{|Q|} \int_{Q}|f(y)| d y
$$

for any $f \in L_{\text {loc }}^{1}(\Omega)$ and $x \in \Omega$. This local maximal operator generalizes that contained in [1] when $\Omega=(0, \infty)$ and $\kappa \leq 3 / 2$, that is, $M_{\text {loc }}^{\kappa} f(x)$ defined in (16). For the (global) Hardy-Littlewood maximal function $M$, some class of weights for the weighted problem were

2000 Mathematics Subject Classification. Primary 42B25; Secondary 35K05.

Key words and phrases. Local maximal operator, Laguerre, heat diffusion semigroup, Laguerre-Riesz transforms, weights.

The first author is supported by UNCPBA (Universidad Nacional del Centro). The second author is supported by UNL(Universidad Nacional del Litoral) and CONICET(Consejo Nacional de Investigaciones Científicas y Técnicas). 
obtained by Carleson and Jones [3], Rubio de Francia [15] and Gatto and Gutiérrez [5], independently. It is worth mentioning that the characterization of the weights $v$ such that the Hardy-Littlewood maximal function is bounded on $L^{p}\left(\boldsymbol{R}^{n}, v(x) d x\right), 1<p<\infty$, as much as the weak- $(1,1)$ boundedness, was done by Muckenhoupt in the celebrated paper [13]. The problem of characterization of the pairs $(u, v)$ for which the Hardy-Littlewood function maps $L^{p}\left(\boldsymbol{R}^{n}, v(x) d x\right)$ into $L^{p}\left(\boldsymbol{R}^{n}, u(x) d x\right)$ was solved by Sawyer in [16]. Now we can state the main theorem

THEOREM 1.1. Let $v$ be a weight in $\Omega$. Let $1 \leq p<\infty$. The following statements are equivalent:

(i) There exists a weight $u$ such that $\mathcal{M}_{\mathcal{F}_{\gamma}} f(x)$ is bounded from $L^{p}(\Omega, v(x) d x)$ to $L^{p}(\Omega, u(x) d x)$ for $p>1$, and from $L^{1}(\Omega, v(x) d x)$ to weak- $L^{1}(\Omega, u(x) d x)$ for $p=1$.

(ii) There exists a weight $u$ such that $\mathcal{M}_{\mathcal{F}_{\gamma}} f(x)$ is bounded from $L^{p}(\Omega, v(x) d x)$ to weak $L^{p}(\Omega, u(x) d x)$.

(iii) $v^{-1 / p} \in L_{\mathrm{loc}}^{p^{\prime}}(\Omega)$ for $p>1$, and $v^{-1} \in L_{\mathrm{loc}}^{\infty}(\Omega)$ for $p=1$ (that is, $v$ is locally bounded from below).

This theorem is applied to study the convergence of the Laguerre Heat diffusion semigroup. More precisely, for $\alpha>-1$, let us consider the problem

$$
\left\{\begin{array}{l}
\frac{\partial u_{\alpha}}{\partial t}=-L u_{\alpha}, \\
u_{\alpha}(x, 0)=f(x) \quad x>0,
\end{array}\right.
$$

where $L=L_{\alpha}$ is the second order differential Laguerre operator defined by

$$
L_{\alpha}=-x \frac{d^{2}}{d x^{2}}-\frac{d}{d x}+\frac{x}{4}+\frac{\alpha^{2}}{4 x}
$$

and, at least formally, $u_{\alpha}(x, t)=T_{t} f(x)=e^{-t L_{\alpha}} f(x)$ for $t>0$.

Let $\mathcal{L}_{n}^{\alpha}(x)$ be the Laguerre function

$$
\mathcal{L}_{n}^{\alpha}(x)=\left(\frac{n !}{n+\alpha+1}\right)^{1 / 2} L_{n}^{\alpha}(x) e^{-x / 2} x^{\alpha / 2},
$$

where $L_{n}^{\alpha}(x)$ is the Laguerre polynomial of degree $n$ given by

$$
L_{n}^{\alpha}(x)=\frac{1}{n !} x^{-\alpha} e^{x}\left(\frac{d^{n}}{d x^{n}}\left(x^{\alpha+n} e^{-x}\right)\right), \quad x>0, \quad n=0,1,2, \ldots .
$$

As is well known, the Laguerre functions $\mathcal{L}_{n}^{\alpha}(x)$ are eigenfuncions of the differential operator $L_{\alpha}$ with eigenvalues $\lambda_{\alpha, n}=-n-(\alpha+1) / 2$. Also they form an orthogonal basis on $L^{2}(0, \infty)$. The operator $L_{\alpha}$ is positive and symmetric on $L^{2}(0, \infty)$.

One of the aim of this paper is to get conditions on a weight $v$ such that

$$
\lim _{t \rightarrow 0} T_{t} f(x)=f(x) \text { for almost all } x>0 \text { and for all } f \in L^{p}((0, \infty), v(x) d x) .
$$


This problem was studied in the classical case, that is when $L$ is the Laplacian operator, in [10] and for $L=\frac{1}{2}(-\Delta+2 x \cdot \nabla)$, the Ohrstein-Uhlenbeck operator, in [8]. As is well known, the a.e. convergence leads to study the behaviour of the maximal operator associated to the heat difussion semigroup $T_{t}$ of $L_{\alpha}$ defined by

$$
W_{\alpha}^{*} f(x):=\sup _{t>0}\left|T_{t} f(x)\right| \quad \text { for all } x>0 .
$$

The maximal operator $W_{\alpha}^{*}$ was studied by Stempak in 1994 for $\alpha \geq 0$ (see [19]), and later on by Macías, Segovia and Torrea for negative values of $\alpha$ in [11] (unweighted case) and in [12] (with power weights). Recently the family of weights was enlarged in [2]. We solved this problem by using a non-constructive method due to J. L. Rubio de Francia. We state it in Theorem 2.5. By using Theorem 1.1 we can show the following.

THEOREM 1.2. Let $1 \leq p<\infty$ and $\alpha \geq 0$. Assume that the weight $v$ satisfies the condition

$$
\left\|v^{-1 / p}(y)\left(\chi_{(0,1)}(y)+e^{-c_{0} y} y^{\alpha / 2} \chi_{[1, \infty)}(y)\right)\right\|_{L^{p^{\prime}}((0, \infty), d y)}=C_{v}<\infty,
$$

where $p^{\prime}=p /(p-1)$ and $c_{0}$ is any positive constant less than $1 / 4$. (The constant $c_{0}$ can be choosed equal to $1 / 16$.) Then there exists a weight $u$ such that $W_{\alpha}^{*} f$ maps $L^{p}((0, \infty), v(x) d x)$ into $L^{p}((0, \infty), u(x) d x)$ for $p>1$, and $L^{1}((0, \infty), v(x) d x)$ into weak- $L^{1}((0, \infty), u(x) d x)$ for $p=1$.

As a consequence, the following result can be proved.

COROLlary 1.3. Let $1 \leq p<\infty$ and $\alpha \geq 0$. If $v$ satisfies (8), then

$$
T_{t} f(x) \rightarrow f(x), \quad \text { as } t \rightarrow 0^{+},
$$

for almost every $x \in(0, \infty)$ and every $f \in L^{p}((0, \infty), v(x) d x)$.

Finally, we apply Theorem 1.2 to get the weighted problem for the Riesz-Laguerre operators $\mathcal{R}_{+}^{\alpha}, \alpha \geq 0$ (see (46)).

THEOREM 1.4. Given $\alpha \geq 0$ and $1 \leq p<\infty$, let $v$ be a weight such that

$$
\left\|v^{-1 / p}(y)\left(\chi_{(0,1)}(y)+e^{-c_{0} y} y^{\alpha / 2+1} \chi_{[1, \infty)}(y)\right)\right\|_{L^{p^{\prime}}((0, \infty), d y)}=C_{v}<\infty,
$$

where $c_{0}$ is a constant less than $1 / 4$. (The constant $c_{0}$ can be choosed equal to $1 / 16$.) Then there exists a weight $u$ such that $\mathcal{R}_{+}^{\alpha} f$ maps $L^{p}((0, \infty), v(x) d x)$ into $L^{p}((0, \infty), u(x) d x)$ for $p>1$, and $L^{1}((0, \infty), v(x) d x)$ into weak- $L^{1}((0, \infty), u(x) d x)$ for $p=1$.

The proof of Theorem 1.1 is contained in Section 2. In Section 3, the proof of Theorem 1.2 and its corollary is given. The proof of Theorem 1.4 is obtained in Section 4 .

2. Local maximal Hardy-Littlewood operators. We shall need the following Whitney type covering Lemma (see [18, p. 167] for details). 
LEMMA 2.1. Given a positive constant $c$, there exists a collection $\mathcal{W}_{c}$ of cubes, say $\mathcal{W}_{c}=\left\{Q_{1}, Q_{2}, \ldots\right\}$, such that

$$
\bigcup_{k} Q_{k}=\Omega=\boldsymbol{R}^{n} \backslash F,
$$

the interiors $Q_{k}^{\circ}$ are pairwise disjoint, and $c \operatorname{diam}\left(Q_{k}\right) \leq \operatorname{dist}\left(Q_{k}, F\right) \leq 2(1+c) \operatorname{diam}\left(Q_{k}\right)$.

DEFINITION 2.2. For a given $p \geq 1$, we say that a non-negative weight $w$ belongs to $L_{\text {loc, }, \mathcal{F}_{\gamma}}^{p}$ if $\int_{Q} w^{p} \leq C_{w, Q}<\infty$ for any cube $Q \in \mathcal{F}_{\gamma}$. Also, we understand that a nonnegative weight $w$ is in $L_{\text {loc }}^{p}(\Omega)$ if $\int_{E} w^{p}<\infty$ for any bounded set $E$ such that $\bar{E} \subset \Omega$.

REMARK 2.3. We note that a weight $w$ is in $L_{\mathrm{loc}}^{p}(\Omega)$ if and only if $w \in L_{\mathrm{loc}, \mathcal{F}_{\gamma}}^{p}$ for any $0<\gamma<1$. In fact, the inclusion $L_{\text {loc }}^{p}(\Omega) \subset L_{\text {loc, } \mathcal{F}_{\gamma}}^{p}$ is clear. On the other hand, for a given bounded set $E$ such that $\bar{E} \subset \Omega$, consider a Whitney type covering for $\Omega$ as in Lemma 2.1 above for $c=2 / \gamma$. Then there exists a positive integer $N$ verifying

$$
\bar{E} \subset \bigcup_{k=1}^{N}\left(2 Q\left(x_{k}, r_{k}\right)\right)^{\circ}=\bigcup_{k=1}^{N}\left(2 Q_{k}\right)^{\circ} .
$$

Here we write the cube $Q(x, 2 r)$ by $2 Q$ when $Q=Q(x, r)$. By (12) we have that

$$
2 r_{k} \leq \frac{\gamma}{2} \operatorname{dist}\left(Q_{k}, F\right) \leq \frac{\gamma}{2} 3 r_{k}+\frac{\gamma}{2} \operatorname{dist}\left(2 Q_{k}, F\right) .
$$

Thus,

$$
2 r_{k}<\frac{\gamma}{2-3 \gamma / 2} \operatorname{dist}\left(2 Q_{k}, F\right)<\gamma \operatorname{dist}\left(2 Q_{k}, F\right) .
$$

Hence $\tilde{Q}_{k}=2 Q_{k} \in \mathcal{F}_{\gamma}$, and consequently

$$
\int_{\bar{E}} w^{p} \leq \sum_{k=1}^{N} \int_{\tilde{Q}_{k}} w(x)^{p} d x<\infty
$$

which implies that $w \in L_{\mathrm{loc}}^{p}(\Omega)$.

The local maximal Hardy-Littlewood operator $M_{\mathrm{loc}}^{\kappa}$ is defined by

$$
M_{\mathrm{loc}}^{\kappa} f(x)=\sup _{0<a<x<b<\kappa a} \frac{1}{b-a} \int_{a}^{b}|f(y)| d y, \quad \kappa=16 .
$$

As a consequence of Theorem 1.1, for $\Omega=(0, \infty)$ we have the following result.

THEOREM 2.4. For a given real number $p, 1 \leq p<\infty$, and $\kappa>1$, the following statements are equivalent:

(i) The weight $v$ satisfies

$$
v^{-1 / p} \in L_{\mathrm{loc}}^{p^{\prime}}(0, \infty) \text { for } p>1, \quad \text { and } \quad v^{-1} \in L_{\mathrm{loc}}^{\infty}(0, \infty) \text { for } p=1 .
$$


(ii) There exists a weight $u$ such that $M_{\mathrm{loc}}^{\kappa} f(x)$ is bounded from $L^{p}((0, \infty), v(x) d x)$ to weak- $L^{p}((0, \infty), u(x) d x)$.

(iii) There exists a weight $u$ such that $M_{\mathrm{loc}}^{\kappa} f(x)$ is bounded from $L^{p}((0, \infty), v(x) d x)$ to $L^{p}((0, \infty), u(x) d x)$ for $p>1$ and from $L^{1}((0, \infty), v(x) d x)$ to weak- $L^{1}((0, \infty)$, $u(x) d x)$ for $p=1$.

Before proving Theorems 1.1 and 2.4, we consider the following version of the Theorem of Rubio de Francia (see, for example, [6, p. 554]).

THEOREM 2.5. Let $(X, \mu)$ a measurable space, $\boldsymbol{B}$ a Banach space and $T$ a sublinear operator $T: \boldsymbol{B} \rightarrow L^{q}(X)$ for some $q<p$. Then the inequality

$$
\left\|\left(\sum_{j}\left|T f_{j}\right|^{p}\right)^{1 / p}\right\|_{L^{q}(X)} \leq C\left(\sum_{j}\left\|f_{j}\right\|_{B}^{p}\right)^{1 / p}
$$

holds if and only if there exists a non-negative weight $u$ such that

$$
\|T f\|_{L^{p}(u)} \leq C\|f\|_{\boldsymbol{B}} .
$$

This theorem suggests the following vector-valued inequality.

Proposition 2.6. Let $p$ be a real number with $1<p<\infty$, and let $\left\{Q_{k}=Q\left(x_{k}, r_{k}\right)\right\}$ be a sequence of cubes satisfying the condition of Lemma 2.1 for $c=1 / \gamma$. If $v^{-1 / p} \in L_{\mathrm{loc}}^{p^{\prime}}(\Omega)$ and $0<q<1<p$, then there exists a positive constant $C_{k, v}$ such that the following inequality holds.

$$
\left\|\left(\sum_{j}\left|\mathcal{M}_{\mathcal{F}_{\gamma}} f_{j}\right|^{p}\right)^{1 / p}\right\|_{L^{q}\left(Q_{k}\right)} \leq C_{k, v}\left(\sum_{j}\left\|f_{j}\right\|_{L^{p}(\Omega, v)}^{p}\right)^{1 / p} .
$$

Proof. By replacing $\gamma / 2$ by $\gamma$ in (13), we clearly have $2 r_{k}<3 \gamma r_{k}+\gamma \operatorname{dist}\left(2 Q_{k}, F\right)$. Since $\gamma<1 / 4$, we get that $\overline{2 Q_{k}} \subset \Omega$. Now we can write

$$
f_{j}=f_{j} \chi_{2} Q_{k}+f_{j} \chi\left(2 Q_{k}\right)^{c}=: f_{j}^{\prime}+f_{j}^{\prime \prime} .
$$

For each $k$, we first estimate $\mathcal{M}_{\mathcal{F}_{\gamma}} f_{j}^{\prime}$ on the cube $Q_{k}$. Since $\mathcal{M}_{\mathcal{F}_{\gamma}}$ is of weak type $(1,1)$, by invoking the Theorem of Kolmogorov for $q<1$ and applying the condition $v^{-1 / p} \in L_{\text {loc }}^{p^{\prime}}(\Omega)$, we have

$$
\begin{aligned}
\left\|\left(\sum_{j}\left|\mathcal{M}_{\mathcal{F}_{\gamma}} f_{j}^{\prime}\right|^{p}\right)^{1 / p}\right\|_{L^{q}\left(Q_{k}\right)} & \leq C\left|Q_{k}\right|^{1 / q-1}\left\|\left(\sum_{j}\left|\mathcal{M}_{\mathcal{F}_{\gamma}} f_{j}^{\prime}\right|^{p}\right)^{1 / p}\right\|_{\text {Weak- } L^{1}\left(Q_{k}, d x\right)} \\
& \leq C\left|Q_{k}\right|^{1 / q-1} \int_{2 Q_{k}}\left(\sum_{j}\left|f_{j}(x)\right|^{p}\right)^{1 / p} v^{1 / p}(x) v^{-1 / p}(x) d x \\
& \leq C\left|Q_{k}\right|^{1 / q-1}\left(\sum_{j}\left\|f_{j}\right\|_{L^{p}(\Omega, v)}^{p}\right)^{1 / p}\left(\int_{2 Q_{k}} v^{-p^{\prime} / p}(x) d x\right)^{1 / p^{\prime}} .
\end{aligned}
$$


Therefore,

$$
\left\|\left(\sum_{j}\left|\mathcal{M}_{\mathcal{F}_{\gamma}} f_{j}^{\prime}\right|^{p}\right)^{1 / p}\right\|_{L^{q}\left(Q_{k}\right)} \leq C_{k, v}\left|Q_{k}\right|^{1 / q-1}\left(\sum_{j}\left\|f_{j}\right\|_{L^{p}(\Omega, v)}^{p}\right)^{1 / p} .
$$

Now we estimate the term dealing with $f_{j}^{\prime \prime}$. Suppose that $x \in Q_{k}$. We have to analyze the expression

$$
\mathcal{M}_{\mathcal{F}_{\gamma}} f^{\prime \prime}(x)=\sup _{Q \in \mathcal{F}_{\gamma}, Q \ni x} \frac{1}{|Q|} \int_{Q \cap\left(2 Q_{k}\right)^{c}}|f(y)| d y .
$$

Let $Q=Q\left(x_{0}, r\right)$ be a cube in $\mathcal{F}_{\gamma}$. Consider a point $y \in Q \cap\left(2 Q_{k}\right)^{c}$. Since $x \in Q_{k}$, we have

$$
2 r_{k} \leq\left|y-x_{k}\right| \leq|y-x|+\left|x-x_{k}\right| \leq 2 r+r_{k} .
$$

Hence, we must have $r_{k} \leq 2 r$, and hence $|2 Q| \geq\left|Q_{k}\right|$. From that, we obtain

$$
\mathcal{M}_{\mathcal{F}_{\gamma}} f^{\prime \prime}(x) \leq \frac{2^{n}}{\left|Q_{k}\right|} \sup _{Q \in \mathcal{F}_{\gamma}, Q \ni x} \int_{Q \cap\left(2 Q_{k}\right)^{c}}|f(y)| d y .
$$

Now, we look for a ball $B_{k}=B\left(x_{k}, R_{k}\right)$ such that its center $x_{k}$ is the same as that of $Q_{k}$, $Q \subset B_{k}$ and $\overline{B_{k}} \subset \Omega$. In view of this, for $y \in Q$, we get from (22) that

$$
\begin{aligned}
\left|y-x_{k}\right| & \leq 2 r+r_{k} \\
& \leq 2 \gamma d(x, \partial F)+r_{k} \\
& \leq 2 \gamma\left|x-x_{k}\right|+2 \gamma d\left(x_{k}, \partial F\right)+r_{k} \\
& \leq(1+2 \gamma) r_{k}+2 \gamma d\left(x_{k}, \partial F\right) \\
& =: R_{k} .
\end{aligned}
$$

We claim that this value $R_{k}$ fulfills our goals. Indeed, the calculations above give us $Q \subset B_{k}$. Also, since $\gamma<1 / 4$, by using (12), we get

$$
R_{k}<\left(\frac{1+2 \gamma}{2}+2\right) \gamma d\left(x_{k}, \partial F\right)<\frac{11}{16} d\left(x_{k}, \partial F\right) .
$$

Then, for $x \in Q_{k}$, it follows that

$$
\bigcup\left\{Q ; Q \in \mathcal{F}_{\gamma}, x \in Q\right\} \subset \overline{B_{k}} \subset \Omega .
$$

Now, from (23) and the fact that $v^{-1 / p} \in L_{\text {loc }}^{p^{\prime}}(\Omega)$, we can write

$$
\begin{aligned}
\mathcal{M}_{\mathcal{F}_{\gamma}} f_{j}^{\prime \prime}(x) & \leq \frac{2^{n}}{\left|Q_{k}\right|} \int_{B_{k}}\left|f_{j}\right| v^{1 / p} v^{-1 / p} \\
& \leq \frac{2^{n}}{\left|Q_{k}\right|}\left(\int_{B_{k}} v^{-p^{\prime} / p}(y) d y\right)^{1 / p^{\prime}}\left\|f_{j}\right\|_{L^{p}(\Omega, v)} \\
& =: C_{k, v}\left|Q_{k}\right|^{-1}\left\|f_{j}\right\|_{L^{p}(\Omega, v)} .
\end{aligned}
$$


Then, it follows

$$
\left(\int_{Q_{k}}\left(\sum_{j}\left|\mathcal{M}_{\mathcal{F}_{\gamma}} f_{j}^{\prime \prime}(x)\right|^{p}\right)^{q / p}\right)^{1 / q} \leq C_{k, v}\left|Q_{k}\right|^{1 / q-1}\left(\sum_{j}\left\|f_{j}\right\|_{L^{p}(\Omega, v)}^{p}\right)^{1 / p} .
$$

Putting (21) and (25) together, we can finally obtain the inequality (20), and the proof is finished.

REMARK 2.7. We note that Proposition 2.6 holds for the local maximal HardyLittlewood operator defined by (16) for any $\kappa>1$. In fact, we take an interval $Q_{k}=Q\left(x_{k}, r_{k}\right)$ verifying Lemma 2.1 in such a way that $\overline{2 Q_{k}} \subset \Omega$ (it is enough to take $c>3 / 2$ ). Then (21) holds for $M_{\mathrm{loc}}^{\kappa} f_{j}^{\prime}(x)$. In order to estimate the expression

$$
M_{\mathrm{loc}}^{\kappa} f_{j}^{\prime \prime}(x)=\sup _{0<a<x<b<\kappa a} \frac{1}{b-a} \int_{(a, b) \cap\left(2 Q_{k}\right)^{c}}|f(y)| d y,
$$

by (22), we clearly have

$$
M_{\mathrm{loc}}^{\kappa} f_{j}^{\prime \prime}(x) \leq \frac{2^{n}}{\left|Q_{k}\right|} \sup _{0<a<x<b<\kappa a} \int_{(a, b) \cap\left(2 Q_{k}\right)^{c}}|f(y)| d y .
$$

But, for $x \in Q_{k}$,

$$
\bigcup\{(a, b) ; a<x<b<\kappa a\} \subset(x / \kappa, \kappa x) \subset\left[\frac{x_{k}-r_{k}}{\kappa}, \kappa\left(x_{k}+r_{k}\right)\right] \subset(0, \infty) .
$$

Therefore, (25) follows for $M_{\mathrm{loc}}^{\kappa} f_{j}^{\prime \prime}(x)$.

Proof OF TheOREM 1.1. First, let us suppose that $v^{-1 / p} \in L_{\text {loc }}^{p^{\prime}}(\Omega)$. By applying Proposition 2.6 above for $0<q<1<p$, we have that the operator $\mathcal{M}_{\mathcal{F}_{\gamma}}$ satisfies the condition of Theorem 2.5 in each set $Q_{k}$. Hence a family of weights $u_{k}$ supported in $Q_{k}$ satisfying (19) in Theorem 2.5 can be found. Thus the weight defined by

$$
u(x):=\sum_{k} 2^{-k p} c_{k}^{-p} u_{k}(x) \chi_{Q_{k}}(x)
$$

satisfies (i) as we wanted.

For the case $p=1$, we use the weak- $(1,1)$ continuity of the Hardy-Littlewood maximal operator, and then

$$
\begin{aligned}
\left|\left\{x \in Q_{k} ; \mathcal{M}_{\mathcal{F}_{\gamma}} f^{\prime}(x)>\lambda\right\}\right| & \leq \frac{C}{\lambda}\left\|f^{\prime}\right\|_{L^{1}}=\frac{C}{\lambda} \int_{2 Q_{k}}|f(x)| d x \\
& \leq \frac{C}{\lambda}\left(\int_{2 Q_{k}}|f(x)| v(x) d x\right)\left\|v^{-1}(\cdot) \chi_{2 Q_{k}}(\cdot)\right\|_{L^{\infty}\left(\boldsymbol{R}^{n}, d x\right)} \\
& \leq \frac{C_{k, v}}{\lambda} \int|f(x)| v(x) d x
\end{aligned}
$$

Pasting (24) and (27) together, we get

$$
\left|\left\{x \in Q_{k} ; \mathcal{M}_{\mathcal{F}_{\gamma}} f(x)>\lambda\right\}\right| \leq \frac{C_{k, v}}{\lambda} \int|f(x)| v(x) d x .
$$


Hence the weight $u(x)=\sum_{k} 1 / 2^{k} C_{k, v} \chi Q_{k}(x)$ gives (ii)

Now, suppose that (ii) holds. Let $Q\left(x_{0}, R\right)$ a fixed cube such that $4 Q\left(x_{0}, R\right) \in \mathcal{F}_{\gamma}$. Since then $Q\left(x_{0}, R\right) \subset Q(x, 2 R)$ for $x \in Q\left(x_{0}, R\right)$ and $Q(x, 2 R) \in \mathcal{F}_{\gamma}$, for any nonnegative $f$, we have

$$
\mathcal{M}_{\mathcal{F}_{\gamma}} f(x) \geq \frac{1}{R^{n}} \int_{Q(x, 2 R)} f(y) d y>\frac{1}{R^{n}} \int_{Q\left(x_{0}, R\right)} f(y) d y, x \in Q\left(x_{0}, R\right) .
$$

Therefore, by (ii) and the Kolmogorov inequality, we have for $s<p$,

$$
\begin{aligned}
\left(\frac{1}{R^{n}} \int_{Q\left(x_{0}, R\right)} f(y) d y\right) & \left(u\left(Q\left(x_{0}, R\right)\right)\right)^{1 / s} \\
& \leq C\left(\int_{Q\left(x_{0}, R\right)} \mathcal{M}_{\mathcal{F}_{\gamma}} f(x)^{s} u(x) d x\right)^{1 / s} \\
& \leq C u\left(Q\left(x_{0}, R\right)\right)^{1 / s-1 / p}\left(\int f^{p}(y) v(y) d y\right)^{1 / p} .
\end{aligned}
$$

Given an arbitrary positive function $g \in L^{p}\left(Q\left(x_{0}, R\right)\right)$, we choose $f=g v^{-1 / p}$, then we have

$$
\left(\frac{1}{R^{n}} \int_{Q\left(x_{0}, R\right)} g(y) v^{-1 / p}(y) d y\right) \leq C u\left(Q\left(x_{0}, R\right)\right)^{-1 / p}\left(\int g^{p}(y) d y\right)^{1 / p} .
$$

By duality we conclude that $v^{-1 / p}$ belongs to $L^{p^{\prime}}\left(Q\left(x_{0}, R\right), d x\right)$. Since any cube $Q \in \mathcal{F}_{\gamma}$ can be covered by a finite family of cubes $Q_{i}$ with the property that $4 Q_{i} \in \mathcal{F}_{\gamma}$, the statement (iii) follows.

Proof OF TheOREM 2.4. We first note that the case $\kappa \leq 3 / 2$ is contained in Theorem 1.1. For any $\kappa>1$, assume first that (iii) holds. Then, the statement (i) follows as in Theorem 1.1 , in view of Remark 2.7. To prove the implication (ii) $\Rightarrow$ (iii), we proceed as in the proof of Theorem 1.1, but taking this time an interval $Q \subset \Omega$ such that $4 Q \in \mathcal{F}_{\kappa}$, where

$$
\mathcal{F}_{\kappa}=\{Q=(a, b) \subset \Omega ; a<b<\kappa a\} .
$$

3. The heat diffusion Laguerre operators. For the heat diffusion semigroup $T_{t}$, we recall the well-known expression

$$
T_{t} f(x)=\int K_{\alpha}(t, x, y) f(y) d y,
$$

where

$$
K_{\alpha}(t, x, y)=W_{\alpha}\left(\frac{1-e^{t / 2}}{1+e^{t / 2}}, x, y\right)
$$

with

$$
W_{\alpha}(s, x, y)=\frac{1-s^{2}}{4 s} e^{-(s+1 / s)\left(x^{1 / 2}-y^{1 / 2}\right)^{2} / 4} e^{-(s+1 / s)(x y)^{1 / 2} / 2} I_{\alpha}\left(\frac{1-s^{2}}{2 s}(x y)^{1 / 2}\right)
$$


after the change of parameters $s=\left(1-e^{-t / 2}\right) /\left(1+e^{-t / 2}\right)$. Here, $I_{\alpha}$ is the modified Bessel function of order $\alpha$, any $\alpha>-1$. In this way, we have

$$
W_{\alpha}^{*} f(x)=\sup _{0<s<1} \int W_{\alpha}(s, x, y) f(y) d y .
$$

The proof of Theorem 1.2 is based on the pointwise estimate (see [2])

$$
W_{\alpha}^{*} f(x) \leq\left(H_{0}^{\alpha / 2}+M_{\mathrm{loc}}^{\kappa}+T^{\alpha / 2}\right) f(x),
$$

where $H_{0}^{\alpha / 2}$ is a Hardy operator modified at the origin

$$
H_{0}^{\alpha / 2} f(x):=x^{-\alpha / 2-1} \int_{0}^{x} f(y) y^{\alpha / 2} d y, \quad \alpha / 2>-1,
$$

and $T^{\alpha / 2}$ is the maximal operator

$$
T^{\alpha / 2} f(x)=\sup _{0<s<1}\left|T_{s}^{\alpha / 2} f(x)\right|,
$$

with $T_{s}^{\alpha / 2}$ the operator given by

$$
\begin{aligned}
T_{s}^{\alpha / 2} f(x) & =x^{\alpha / 2} \int_{x}^{\infty}\left[\left(\frac{y}{s}\right)^{\alpha+1} e^{-c_{0} y / s}\right] y^{-\alpha / 2-1} f(y) d y \\
& =: x^{\alpha / 2} \int_{x}^{\infty} \phi(s, y) y^{-\alpha / 2-1} f(y) d y .
\end{aligned}
$$

Here $c_{0}$ is equal to $1 / 16$. Besides, we have implicitly defined

$$
\phi(s, y)=\left(\frac{y}{s}\right)^{\alpha+1} e^{-c_{0} y / s} .
$$

REMARK 3.1. We note that the constant $c_{0}$ is not optimal. This depends on the local region considered. It is not hard to check that $c_{0}$ tends to $1 / 4$ when $\kappa$ tends to $\infty$. The constant $c_{0}=1 / 16$ is obtained by considering in [2, pp. 343] the local region defined by

$$
\frac{x}{4}<y<4 x
$$

where $\kappa=16$. In this way, for the global case at infinity $4 x<y<\infty$, we have

$$
e^{-\left|x^{1 / 2}-y^{1 / 2}\right|^{2} / 4 s} \leq e^{-y / 16 s} \equiv e^{-c_{0} y / s}
$$

whose right-hand side appeared in (32).

In view of (30), we devote the rest of this section to solve the weighted problem for the operators $H_{0}^{\alpha} f$ and $T^{\alpha / 2} f$. Since the operators $T^{\alpha / 2}, \alpha \geq 0$, are pointwise bounded by Hardy type modified operators at the infinity, they are of weak type $(1,1)$ (see [1]). Therefore, we can proceed in a similar way as in Proposition 2.6, obtaining the following Proposition.

Proposition 3.2. Let $1 \leq p<\infty$ and $\alpha \geq 0$. Assume that the weight $v$ satisfies

$$
\left\|v^{-1 / p}(y)\left(\chi_{(0,1)}(y)+e^{-c_{0} y} y^{\alpha / 2} \chi_{[1, \infty)}(y)\right)\right\|_{L^{p^{\prime}}((0, \infty), d y)}=C_{v}<\infty .
$$


Then, for $0<q<1<p$,

$$
\left\|\left(\sum_{j}\left|T^{\alpha / 2} f_{j}\right|^{p}(\cdot)\right)^{1 / p}\right\|_{L^{q}((0, \infty) d x)} \leq C\left(\sum_{j}\left\|f_{j}\right\|_{L^{p}((0, \infty), v(x) d x)}^{p}\right)^{1 / p} .
$$

Moreover, there exists a weight $u$ such that $T^{\alpha / 2} f(x)$ is bounded from $L^{p}((0, \infty), v(x) d x)$ to $L^{p}((0, \infty), u(x) d x)$ for $p>1$, and from $L^{1}((0, \infty), v(x) d x)$ to weak- $L^{1}((0, \infty), u(x) d x)$ for $p=1$.

Proof. Before starting, we set the constant

$$
A:=\frac{\alpha+1}{c_{0}} .
$$

Let $\left\{Q_{k}\right\}_{k \geq 1}$ be the sequence of intervals $Q_{k}=\left(A^{k}, A^{k+1}\right]$ for $k \geq 1$, and denote $Q_{0}=$ $(0, A]$. Also, let us write

$$
f_{j}=f_{j} \chi_{\left(0, A^{k+1}\right]}+f_{j} \chi_{\left(A^{k+1}, \infty\right)}=: f_{j}^{\prime}+f_{j}^{\prime \prime} .
$$

Proceeding as in Proposition 2.6, we can see that

$$
\begin{aligned}
& \left\|\left(\sum_{j}\left|T^{\alpha / 2} f_{j}^{\prime}\right|^{p}\right)^{1 / p}\right\|_{L^{q}\left(Q_{k}\right)} \\
& \quad \leq C\left|Q_{k}\right|^{1 / q-1}\left(\sum_{j}\left\|f_{j}\right\|_{L^{p}((0, \infty), v)}^{p}\right)^{1 / p}\left(\int_{\left(0, A^{k+1}\right]} v^{-p^{\prime} / p}(x) d x\right)^{1 / p^{\prime}} \\
& \quad \leq C_{k, v}^{\prime}\left|Q_{k}\right|^{1 / q}\left(\sum_{j}\left\|f_{j}\right\|_{L^{p}((0, \infty), v)}^{p}\right)^{1 / p}
\end{aligned}
$$

where

$$
C_{k, v}^{\prime}:=\left|Q_{k}\right|^{-1}\left(\int_{\left(0, A^{k+1}\right]} v^{-p^{\prime} / p}(x) d x\right)^{1 / p^{\prime}} .
$$

Now, we analyze $T^{\alpha / 2} f_{j}^{\prime \prime}(x)$ for $x \in Q_{k}$. The function $\phi(s, y)$ (see equation (33)) is increasing in the variable $s$ whenever $y \geq A$; therefore, it attains its maximum at $s=1$. Finally, with the aid of Hölder inequality, we have

$$
\begin{aligned}
T^{\alpha / 2} f_{j}^{\prime \prime}(x) & \leq \sup _{0<s<1} A^{(k+1) \alpha / 2} \int_{A^{k+1}}^{\infty} \phi(s, y) y^{-\alpha / 2-1} f_{j}(y) d y \\
& \leq C_{k, v}^{\prime \prime}\|f\|_{L^{p}((0, \infty), v(x) d x)}
\end{aligned}
$$

where

$$
C_{k, v}^{\prime \prime} \doteq\left(A^{p^{\prime}(k+1) \alpha / 2} \int_{1}^{\infty} e^{-p^{\prime} c_{0} y} y^{p^{\prime} \alpha / 2} v^{-p^{\prime} / p}(y) d y\right)^{1 / p^{\prime}}<\infty .
$$

By denoting $C_{k, v}=C_{k, v}^{\prime}+C_{k, v}^{\prime \prime}$, we clearly have

$$
C_{k, v} \leq C_{k}\left(\int_{0}^{\infty} v^{-p^{\prime} / p}(y)\left(\chi_{(0,1)}+e^{-p^{\prime} c_{0} y} y^{p \alpha / 2} \chi_{[1, \infty)}(y)\right) d y\right)^{1 / p^{\prime}} .
$$


Putting (37) and (38) together, we see that the operator satisfies the condition of Theorem 2.5 on each set $Q_{k}$. Therefore, as before, a family of weights $u_{k}$ supported in $Q_{k}$ satisfying the statement (19) can be found. Finally, we define

$$
u(x)=\sum_{k=0}^{\infty} 2^{-k p} c_{k}^{-p} u_{k}(x) \chi Q_{k}(x)
$$

which complete the proof when $1<p$. For the case $p=1$, using the weak- $(1,1)$ continuity of the operator $T^{\alpha / 2} f(x)$ and proceeding as the corresponding bound of $\mathcal{M}_{\mathcal{F}_{\gamma}}$, we have

$$
\begin{aligned}
\mid\{x & \left.\in Q_{k} ; T^{\alpha / 2} f^{\prime}(x)>\lambda\right\}\left|\leq \frac{C}{\lambda}\left\|f^{\prime}\right\|_{L^{1}}=\frac{C}{\lambda} \int_{2 Q_{k}}\right| f(x) \mid d x \\
& \leq \frac{C}{\lambda}\left(\int_{2 Q_{k}}|f(x)| v(x) d x\right)\left\|v^{-1}(\cdot) \chi_{2 Q_{k}}(\cdot)\right\|_{L^{\infty}\left(\boldsymbol{R}^{n}, d x\right)} \\
& \leq \frac{C_{k, v}^{\prime}}{\lambda} \int|f(x)| v(x) d x .
\end{aligned}
$$

From(40) and (38), by taking

$$
C_{k, v}^{\prime \prime} \doteq A^{(k+1) \alpha / 2}\left\|e^{-c_{0} y} y^{\alpha / 2} v^{-1 / p}(y) \chi_{[1, \infty)}(y)\right\|_{L^{\infty}((0, \infty), d y)} \text { and } C_{k, v}=C_{k, v}^{\prime}+C_{k, v}^{\prime \prime},
$$

we get

$$
\left|\left\{x \in Q_{k} ; T^{\alpha / 2} f(x)>\lambda\right\}\right| \leq \frac{C_{k, v}}{\lambda} \int|f(x)| v(x) d x .
$$

Hence the weight $u(x)=\sum_{k} 2^{-k p} C_{k, v}^{-1} \chi Q_{k}(x)$ gives the conclusion.

Now, pay attention to the Hardy operators. Since $H_{0}^{\alpha / 2}$ has weak type $(1,1)$ whenever $\alpha \geq 0$ (see [1]), we can apply similar procedures as for $T^{\alpha / 2}$. Again, for $Q_{k}=\left(A^{k}, A^{k+1}\right.$ ] we take $f_{j}=: f_{j}^{\prime}+f_{j}^{\prime \prime}$, with $f_{j}^{\prime}$ supported in $\left(0, A^{k+1}\right]$ and $f_{j}^{\prime \prime}$ supported in $\left(A^{k+1}, \infty\right)$. However, observe that $H_{0}^{\alpha / 2} f_{j}^{\prime \prime}(x)=0$ because $f_{j}^{\prime \prime}(x)$ is zero in the domain of integration in (31). For that reason, it is enough to look only through the term $H_{0}^{\alpha / 2} f_{j}^{\prime}(x)$. But, since $H_{0}^{\alpha / 2}$ has weak type $(1,1)$, we can follow the same lines as before, obtaining the next result.

PROPOSITION 3.3. Let $1 \leq p<\infty$ and $\alpha \geq 0$. Let $v$ be a weight satisfying

$$
v^{-1 / p} \in L_{\mathrm{loc}}^{p^{\prime}}(0, \infty) \text {. }
$$

If $0<q<1<p$, then

$$
\left\|\left(\sum_{j}\left|H_{0}^{\alpha / 2} f_{j}\right|^{p}(\cdot)\right)^{1 / p}\right\|_{L^{q}((0, \infty), d x)} \leq C\left(\sum_{j}\left\|f_{j}\right\|_{L^{p}((0, \infty), v)}^{p}\right)^{1 / p} .
$$

Moreover, there exists a weight $u$ such that $H_{0}^{\alpha / 2} f(x)$ is bounded from $L^{p}((0, \infty), v(x) d x)$ to $L^{p}((0, \infty), u(x) d x)$ for $p>1$, and from $L^{1}((0, \infty), v(x) d x)$ to weak- $L^{1}((0, \infty), u(x) d x)$ for $p=1$. 
Proof of Theorem 1.2. By taking $\alpha \geq 0$, applying (30), Theorem 2.4 and Propositions 3.2 and 3.3, we prove Theorem 1.2, as desired.

Proof of Corollary 1.3. Since the family $\left\{e^{-t L_{\alpha}}\right\}$ is a symmetric diffusion semigroup for $\alpha \geq 0$, we deduce (see [17])

$$
T_{t} f(x) \rightarrow f(x), \quad \text { as } t \rightarrow 0,
$$

for all $f \in L^{2}(0, \infty)$, in particular for any $f$ in the set $\mathcal{C}_{0}$ consisting of all continuous functions on $(0, \infty)$ with compact support, which is dense in $L^{p}(v)$ for all $1 \leq p<\infty$. Finally, by using Theorem 1.2, we have that $T_{t} f(x) \rightarrow f(x)$ as $t \rightarrow 0$, almost every $x \in(0, \infty)$, for all $f \in L^{p}((0, \infty), v)$ with $1 \leq p<\infty$.

4. Riesz-Laguerre operators. In this Section we study the weighted problem for the Riesz-Laguerre operators $\mathcal{R}^{\alpha}, \alpha \geq 0$. Their properties are studied in [7] and [9]. For the self-adjoint differential operator $L_{\alpha}$, we shall consider the Riesz-potentials (see [20])

$$
L^{-\sigma} f(x)=\frac{1}{\Gamma(\sigma)} \int_{0}^{\infty} T_{t} f(x) t^{\sigma-1} d t, \sigma>0,
$$

where $T_{t}=e^{-t L_{\alpha}}$ is the heat diffusion semigroup. In order to define the corresponding Riesz transforms, the following first order derivatives can be considered (see [9]).

$$
\delta^{\alpha}=\sqrt{x} \frac{d}{d x}+\frac{1}{2}\left(\sqrt{x}-\frac{\alpha}{\sqrt{x}}\right) \quad \text { and } \quad \partial^{\alpha+1}=-\sqrt{x} \frac{d}{d x}+\frac{1}{2}\left(\sqrt{x}-\frac{\alpha+1}{\sqrt{x}}\right),
$$

which satisfies

$$
L_{\alpha}-\left(\frac{\alpha+1}{2}\right)=\left(\delta_{y}^{\alpha}\right)^{*} \delta_{y}^{\alpha}=\partial_{y}^{\alpha+1} \delta_{y}^{\alpha}, \quad L_{\beta}-\left(\frac{\beta-1}{2}\right)=\left(\partial_{y}^{\beta}\right)^{*} \partial_{y}^{\beta}=\delta_{y}^{\beta-1} \partial_{y}^{\beta} .
$$

Hence the Riesz transforms for the Laguerre function expansions are defined by

$$
\mathcal{R}_{+}^{\alpha}=\delta_{y}^{\alpha}\left(L_{\alpha}\right)^{-1 / 2}, \quad \alpha>-1, \quad \text { and } \quad \mathcal{R}_{-}^{\beta}=\partial_{y}^{\beta}\left(L_{\beta}\right)^{-1 / 2}, \quad \beta>0 .
$$

For $f, g \in C_{c}^{\infty}(0, \infty)$ with disjoint supports, the following equality holds

$$
\left\langle\mathcal{R}_{(+,-)}^{\alpha} f, g\right\rangle=\int_{(0, \infty)} \int_{(0, \infty)} \mathcal{R}_{(+,-)}^{\alpha}(x, z) f(x) g(z) d x d z .
$$

We shall only consider the weighted problem for the Riesz-Laguerre transform $R_{+}^{\alpha}$. To get the kernel of $R_{+}^{\alpha}$, we write

$$
\begin{aligned}
\mathcal{R}_{+}^{\alpha}(x, z)= & C\left(\sqrt{x} \frac{d}{d x}+\frac{1}{2}\left(\sqrt{x}-\frac{\alpha}{\sqrt{x}}\right)\right)\left(L^{\alpha}\right)^{-1 / 2}(x, z) \\
= & C \int_{0}^{1}\left(\frac{1}{2} \frac{1-s^{2}}{2 s} z^{1 / 2} W_{\alpha+1}(s, x, z)-\frac{1}{2} \frac{(1-s)^{2}}{2 s} x^{1 / 2} W_{\alpha}(s, x, z)\right) \\
& \quad \times\left(\log \frac{1+s}{1-s}\right)^{-1 / 2} \frac{d s}{1-s^{2}} \\
= & C \int_{0}^{1} R_{\alpha}(x, z, s)\left(\log \frac{1+s}{1-s}\right)^{-1 / 2} \frac{d s}{1-s^{2}}
\end{aligned}
$$


where $s=\left(1-e^{-t / 2}\right) /\left(1+e^{-t / 2}\right)$ and $W_{\alpha}(s, x, z)$ is the heat-diffusion kernel given by (29). where

Proof of THEOREM 1.4. We will use the decomposition $\mathcal{R}_{+}^{\alpha}=: \mathcal{R}_{+, \text {loc }}^{\alpha}+\mathcal{R}_{+, \text {glob }}^{\alpha}$,

$$
\mathcal{R}_{+, \text {glob }}^{\alpha} f(x):=\int_{0}^{\infty} \mathcal{R}_{+}^{\alpha}(x, z) \chi_{\{z \leq x / 4\} \cup\{z \geq 4 x\}} f(z) d z .
$$

It is shown in [7] that $\mathcal{R}_{+, \text {loc }}^{\alpha}$ is a local Calderón-Zygmund operator for $\alpha>-1$. Consequently, as is proved in [14], $\mathcal{R}_{+, \text {loc }}^{\alpha}$ is strong type $(p, p)$ for any $1<p<\infty$ and weak type $(1,1)$. Now, for a function $f$ in $L^{p}((0, \infty), v(x) d x), p>1$, we can write $f=: f_{k}^{\prime}+f_{k}^{\prime \prime}$, by setting $f_{k}^{\prime}=f \chi_{\left[0,2^{k+3}\right)}$. First, in the case that $f \in C_{c}^{\infty}(0, \infty)$ and $x \in Q_{k}=\left(2^{k}, 2^{k+1}\right]$, we have

$$
\mathcal{R}_{+, \text {loc }}^{\alpha} f_{k}^{\prime \prime}(x)=\int_{x / 4}^{4 x} \mathcal{R}^{\alpha}(x, z) f_{k}^{\prime \prime}(z) d z=0 .
$$

For $\mathcal{R}_{+, \text {loc }}^{\alpha} f_{k}^{\prime}$ we can proceed in the same way as in Proposition 3.2. Applying the Theorem of Rubio de Francia and the weak type $(1,1)$ of $\mathcal{R}_{+, \text {loc }}^{\alpha}$, we can conclude that

$$
\left\|\mathcal{R}_{+, \text {loc }}^{\alpha} f\right\|_{L^{p}((0, \infty), u(x) d x)} \leq C\|f\|_{L^{p}((0, \infty), v(x) d x)}
$$

for all weight $v$ satisfying $v^{-1 / p} \in L_{\text {loc }}^{p^{\prime}}(0, \infty)$. Now we turn to $\mathcal{R}_{+, \text {glob }}^{\alpha}$. By the estimate obtained in [7, pp. 16-17], for $f \geq 0$, we have

$$
\mathcal{R}_{+, \text {glob }}^{\alpha} f(x) \leq C x^{-1 / 2} \tilde{W}_{\alpha+1, \text { glob }}^{*}\left(z^{1 / 2} f\right)(x)+C \tilde{W}_{\alpha, \text { glob }}^{*} f(x)=: J_{1}+J_{2} .
$$

Here, $\tilde{W}_{\alpha, \text { glob }}^{*}$ is defined by

$$
\tilde{W}_{\alpha, \text { glob }}^{*} f(x):=\sup _{0<s \leq 1} \int \tilde{W}_{\alpha}(s, x, z) f(z) d z,
$$

where $\widetilde{W}_{\alpha}^{*}$ is a minor modification of $W_{\alpha}^{*}$, the maximal operator. More precisely,

$$
\widetilde{W}_{\alpha}^{*} f(x)=\sup _{0<s \leq 1} \int \widetilde{W}_{\alpha}(x, z, s) f(z) d y,
$$

where $\widetilde{W}_{\alpha}=\tilde{M} I_{\alpha}$ with

$$
\tilde{M}=\frac{1}{2} \frac{1-s^{2}}{2 s} e^{-(s+1 / s)\left|x^{1 / 2}-z^{1 / 2}\right|^{2} / 8} e^{-(s+1 / s)(x z)^{1 / 2} / 2} .
$$

Therefore, the only difference with $W_{\alpha}$ is the $1 / 8$ in the first exponential instead of $1 / 4$. We refer to [2] in order to check that such a change does not affect the estimate obtained there

$$
J_{2}:=\sup _{0<s<1} \int \widetilde{W}_{\alpha}(s, x, z) \chi_{G}(z) f(z) d z \leq C\left(H_{0}^{\alpha / 2} f(x)+\sup _{0<s<1}\left|T_{s}^{\alpha / 2} f(x)\right|\right)
$$

for all $0<s<1$, where $\chi_{G}(z)=\chi_{\{z \leq x / 4\} \cup\{z \geq 4 x\}}(z)$ and $\sup _{0<s<1}\left|T_{s}^{\alpha / 2} f(x)\right|$ is like in (32) with $c_{0}$ replaced by $c_{0} / 2$. For $J_{1}$, we clearly have

$$
J_{1}=C x^{-1 / 2} \sup _{0<s \leq 1} \int \widetilde{W}_{\alpha+1}(s, x, z) \chi_{G}(z) z^{1 / 2} f(z) d z \leq H_{0}^{(\alpha+2) / 2} f(x)+\tilde{T}^{\alpha / 2} f(x)
$$


where

$$
\tilde{T}^{\alpha / 2} f(x):=\sup _{0<s \leq 1} x^{\alpha / 2} \int_{4 x}^{\infty} z^{-\alpha / 2-1} \tilde{\phi}(s, z) f(z) d z
$$

with

$$
\tilde{\phi}(s, z)=\left(\frac{z}{s}\right)^{\alpha+2} e^{-\left(c_{0} / 2\right) z / s} .
$$

The function $\tilde{\phi}(s, y)$ satisfies the properties (3.1), (3.2) and (3.3) listed in [2, p. 336]. So the operator $\tilde{T}^{\alpha / 2}$ is of weak type $(1,1)$ and strong type $(p, p)$ for any $1<p<\infty$. Summing up, the inequality

$$
\mathcal{R}_{+, \text {glob }}^{\alpha} f(x) \leq C\left(H_{0}^{(\alpha+1) / 2}+\tilde{T}^{\alpha / 2}+H_{0}^{\alpha / 2}+T^{\alpha / 2}\right) f(x)
$$

holds for $f \geq 0$ and for all $x>0$.

Now, by applying Proposition 3.2, but this time taking into account the particular expression of the kernel $\tilde{\phi}(s, y)$, and Proposition 3.3, we get the conclusion of Theorem 1.4 .

\section{REFERENCES}

[1] K. Andersen And B. Muckenhoupt, Weighted weak type Hardy inequalities with applications to Hilbert transforms and maximal functions, Studia Math. 72 (1982), 9-26.

[2] A. Chicco Ruiz And E. HARboure, Weighted norm inequalities for heat-diffusion Laguerre's semigroups, Math. Z. 57 (2007), no. 2, 329-354.

[ 3 ] L. Carleson And P. Jones, Weighted norm inequalities and a Theorem of Koosis, Mittag-Leffler Institut, Report n. 2, 1981.

[4] C.-C. Lin And K. Stempak, Local Hardy-Littlewood maximal operator, Math. Ann. 348 (2010), no. 4, 797-813.

[ 5 ] A. Gatto And C. GutiÉrRez, On weighted norm inequalities for the maximal function, Studia Math. 76 (1983), 59-62.

[6] J. García-Cuerva ANd J. Rubio de Francia, Weighted norm inequalities and related topics, NorthHolland Math. Stud. 116, North-Holland Publishing Co., Amsterdam, 1985.

[ 7 ] E. Harboure, C. Segovia, J. Torrea and B. Viviani, Power weighted $L^{p}$-inequalities for LaguerreRiesz transforms, Ark. Mat. 46 (2008), no. 2, 285-313.

[ 8 ] E. Harboure, J. Torrea And B. Viviani, On the search for weighted inequalities for operators related to the Ornstein-Uhlenbeck semigroup, Math. Ann. 318 (2000), no. 2, 341-353.

[ 9 ] E. Harboure, J. Torrea And B. Viviani, Riesz transforms for Laguerre expansions, Indiana Univ. Math. J. 55 (2006), no. 3, 999-1014.

[10] S. Hartzstein, J. Torrea And B. Viviani, A note on the convergence to initial data of heat and Poisson equations, Proc. Amer. Math. Soc. 141 (2013), no. 4, 1323-1333.

[11] R. Macías, C. Segovia And J. TorRea, Heat-diffusion maximal operators for Laguerre semigroups with negative parameters, J. Funct. Anal. 229 (2005), no. 2, 300-316.

[12] R. Macías, C. Segovia And J. Torrea, Weighted norm estimates for the maximal operator of the Laguerre functions heat diffusion semigroup, Studia Math. 172 (2006), no. 2, 149-167.

[13] B. Muckenhoupt, Weighted norm inequalities for the Hardy maximal function, Trans. Amer. Math. Soc. 165 (1972), 207-226. 
[14] A. NOWAK AND K. STEMPAK, Weighted estimates for the Hankel transform transplantation operator, Tohoku Math. J. (2) 58 (2006), no. 2, 227-301.

[15] J. Rubio DE FrAnCIA, Weighted norm inequalities and vector valued inequalities, Harmonic analysis (Minneapolis, Minn., 1981), pp. 86-101, Lecture Notes in Math. 908, Springer, Berlin-New York, 1982.

[16] E. SAWYER, Two weight norm inequalities for certain maximal and integral operators, Harmonic analysis (Minneapolis, Minn., 1981), pp. 102-127, Lecture Notes in Math. 908, Springer, Berlin-New York, 1982.

[17] E. STEIn, Topics in harmonic analysis related to the Littlewood-Paley theory, Annals of Mathematics Studies, No. 63, Princeton University Press, Princeton, N.J.; University of Tokyo Press, Tokyo, 1970.

[18] E. STEIn, Singular integrals and differentiability properties of functions, Princeton Mathematical Series, No. 30, Princeton Univ. Press, Princeton, N.J., 1970.

[19] K. StemPaK, Heat-diffusion and Poisson integrals for Laguerre expansions, Tohoku Math. J. (2) 46 (1994), no. $1,83-104$.

[20] S. Thangavelu, Lectures on Hermite and Laguerre expansions, Math. Notes 42, Princeton University Press, Princeton, NJ, 1993.

\author{
NUCOMPA, NÚCLEO CONSO-LIDADO DE \\ Matemática PURA Y APLICADA \\ UNIVERSIDAD NACIONAL DEL CENTRO \\ PINTO 399, 7000 TANDIL \\ ARgEnTINA \\ E-mail address: pablosebviola@yahoo.com.ar
}

IMAL, INSTITUTO DE MATEMÁticA

APLICADA DEL LITORAL

CONICET - UNIVERSIDAD NACIONAL DEL

LITORAL

GÜEMES 3450, 3000 SANTA FE

ARGENTINA

E-mail address: beatriz.viviani@gmail.com 